\title{
OPEN Clinical features and disease severity in an Iranian population of inpatients with COVID-19
}

\begin{abstract}
Shima Nabavi ${ }^{1}$, Zahra Javidarabshahi ${ }^{2}$, Abolghasem Allahyari ${ }^{3}$, Mohammad Ramezani ${ }^{4}$, Mohsen Seddigh-Shamsi ${ }^{3}$, Sahar Ravanshad ${ }^{1}$, Mina AkbariRad ${ }^{1}$, Farnoosh Ebrahimzadeh ${ }^{1}$, Shohre Khatami ${ }^{1}$, Maryam Emadzadeh ${ }^{5}$, Neda Saeedian ${ }^{1}$, Ahmadreza Zarifian ${ }^{5}$, Maryam Miri', Fariba Rezaeetalab ${ }^{2}$, Sepide Hejazi², Reza Basiri² \& Mahnaz Mozdourian ${ }^{2}$
\end{abstract}

Coronavirus disease 2019 (COVID-19) can present with a variety of symptoms. Severity of the disease may be associated with several factors. Here, we review clinical features of COVID-19 inpatients with different severities. This cross-sectional study was performed in Imam Reza hospital, Mashhad, Iran, during February-April 2020. COVID-19 patients with typical computed tomography (CT) patterns and/ or positive reverse-transcriptase polymerase chain reaction (RT-PCR) were included. The patients were classified into three groups of moderate, severe, and critical based on disease severity. Demographic, clinical, laboratory, and radiologic findings were collected and compared. $\mathrm{P}<0.05$ was considered statistically significant. Overall, 200 patients with mean age of $69.75 \pm 6.39$ years, of whom 82 (41\%) were female were studied. Disease was severe/critical in the majority of patients $(167,83.5 \%)$. Disease severity was significantly associated with age, malignant comorbidities, dyspnea, nausea/vomiting, confusion, respiratory rate, pulse rate, $\mathrm{O}_{2}$ saturation, extent of $\mathrm{CT}$ involvement, serum $\mathrm{C}$-reactive protein $(\mathrm{CRP}), \mathrm{pH}_{1} \mathrm{pO}_{2}$, and aspartate transaminase $(\mathrm{P}<0.05)$. Moreover, complications including shock, coagulopathy, acidosis, sepsis, acute respiratory distress syndrome (ARDS), intensive care unit (ICU) admission, and intubation were significantly higher in patients with higher severities $(P<0.05)$. $\mathrm{O}_{2}$ saturation, nausea/vomiting, and extent of lung CT involvement were independent predictors of severe/critical COVID-19 (OR 0.342, 45.93, and 25.48, respectively; $\mathrm{P}<0.05$ ). Our results indicate $\mathrm{O}_{2}$ saturation, nausea/vomiting, and extent of lung $C T$ involvement as independent predictors of severe COVID-19 conditions. Serum CRP levels and $\mathrm{pO}_{2}$ were also considerably higher patients with higher severity and can be used along with other factors to predict severe disease in COVID-19 patients.

In December 2019, a novel coronavirus emerged in Wuhan city of Hubei Province in China ${ }^{1}$. The virus mainly involved the lungs, leading to a severe acute respiratory syndrome; thus it was initially named as severe acute respiratory syndrome coronavirus 2 (SARS-CoV-2). The world health organization (WHO) named the condition as coronavirus disease of 2019 (COVID-19) and announced it as a global health emergency, which soon was recognized a pandemic ${ }^{2}$.

Compared to the two previously known diseases caused by coronaviruses, namely Middle East Respiratory Syndrome (MERS) and Severe Acute Respiratory Syndrome (SARS), COVID-19 has shown higher estimated infectivity rates ( 3.5 vs. 0.92 and 3 infections per infected persons, respectively) and thus can be potentially more contagious $^{3-5}$. However, the disease appears to be less lethal than was the case in SARS and MERS ${ }^{6}$. It seems that most of COVID-19 cases develop a mild or even asymptomatic disease. Other patients mostly show signs and symptoms of a mild to moderate upper respiratory tract illness. However, some cases develop severe pneumonia that is accompanied by respiratory failure and even death ${ }^{7,8}$.

Previous studies on Chinese population have reported different underlying diseases and demographic factors to be associated with further deterioration of the condition of COVID-19 patients and worse outcomes ${ }^{8-10}$. Old

\footnotetext{
${ }^{1}$ Department of Internal Medicine, Faculty of Medicine, Mashhad University of Medical Sciences, Mashhad, Iran. ${ }^{2}$ Lung Diseases Research Center, Mashhad University of Medical Sciences, Mashhad, Iran. ${ }^{3}$ Department of Hematology-Oncology, Faculty of Medicine, Mashhad University of Medical Sciences, Mashhad, Iran. ${ }^{4}$ Student Research Committee, Faculty of Medicine, Mashhad University of Medical Sciences, Mashhad, Iran. ${ }^{5} \mathrm{Clinical}$ Research Development Unit, Ghaem Hospital, Mashhad University of Medical Sciences, Mashhad, Iran. ${ }^{6}$ Kidney Transplantation Complications Research Centre, Mashhad University of Medical Sciences, Mashhad, Iran. ${ }^{\circledR}$ email: mozdorianmh@mums.ac.ir
} 


\begin{tabular}{|l|l|l|l|r|r|r|}
\hline \multirow{2}{*}{ Feature } & Disease severity & \multicolumn{2}{l|}{} \\
\cline { 2 - 6 } & Mild/moderate (N=33) & Severe (N=139) & Critical (N=28) & P $_{1}$ & P $_{2}^{* * *}$ \\
\hline Demographic data & $15(45.5)$ & $55(39.6)$ & $12(42.9)$ & $0.569^{*}$ & 0.807 \\
\hline Sex (female) & $54.87 \pm 18.35$ & $57.80 \pm 14.80$ & $66.71 \pm 16.61$ & $0.149^{* *}$ & $\mathbf{0 . 0 0 9}$ \\
\hline Age (years) & $6(18.2)$ & $43(30.9)$ & $12(42.9)$ & $0.093^{*}$ & 0.111 \\
\hline Comorbid conditions & $5(15.2)$ & $22(15.8)$ & $7(25.0)$ & $0.757^{*}$ & 0.476 \\
\hline DM & $7(21.2)$ & $40(28.8)$ & $10(35.7)$ & $0.310^{*}$ & 0.454 \\
\hline IHD & $0(0.0)$ & $5(3.6)$ & $0(0.0)$ & $0.314^{*}$ & 0.325 \\
\hline Hypertension & $1(3.0)$ & $3(2.2)$ & $1(3.6)$ & $>0.999^{*}$ & 0.888 \\
\hline Asthma & $0(0.0)$ & $2(1.4)$ & $1(3.6)$ & $0.438^{*}$ & 0.517 \\
\hline Autoimmune disease & $1(3.0)$ & $0(0.0)$ & $0(0.0)$ & $0.024^{*}$ & 0.079 \\
\hline CKD & $2(6.1)$ & $13(9.4)$ & $1(3.6)$ & $0.653^{*}$ & 0.721 \\
\hline Transplantation & $0(0.0)$ & $3(2.2)$ & $0(0.0)$ & $0.438^{*}$ & 0.513 \\
\hline COPD & $0(0.0)$ & $2(1.4)$ & $0(0.0)$ & $0.528^{*}$ & 0.642 \\
\hline Cerebrovascular disease & $1(3.0)$ & $0(0.0)$ & $0(0.0)$ & $0.024^{*}$ & 0.079 \\
\hline CNS disease & $0(0.0)$ & $2(1.4)$ & $1(3.6)$ & $0.438^{*}$ & 0.517 \\
\hline Hepatitis & $0(0.0)$ & $3(2.2)$ & $5(17.9)$ & $0.199^{*}$ & $<\mathbf{0 . 0 0 1}$ \\
\hline Hypothyroidism & $5(15.2)$ & $14(10.1)$ & $4(14.3)$ & $0.822^{*}$ & 0.544 \\
\hline Malignancy & $0(0.0)$ & $5(3.6)$ & $0(0.0)$ & $0.593^{*}$ & 0.325 \\
\hline Smoking & $0(0.0)$ & $1(0.7)$ & $0(0.0)$ & $>0.999^{*}$ & 0.802 \\
\hline Addiction & $7.09 \pm 4.31$ & $6.81 \pm 3.54$ & $0.294^{* *}$ & 0.555 \\
\hline Alcohol use & $7.27 \pm 3.39$ & $7.56 \pm 4.74$ & $0.249^{* *}$ & 0.484 \\
\hline Clinical characteristics of COVID-19 & $6.00 \pm 6.50$ & &
\end{tabular}

Table 1. Demographic data and comorbid conditions in patients with different disease severities. DM diabetes mellitus, $I H D$ ischemic heart disease, $C K D$ chronic kidney disease, COPD chronic obstructive pulmonary disease, CNS central nervous system. $\mathrm{P}_{1}$ : Comparison between mild/moderate and severe/critical groups. $\mathrm{P}_{2}$ : Comparison between mild/moderate, severe, and critical groups. Bold values are statistically significant $(\mathrm{P}<0.05) .{ }^{\star}$ Chi-square or Fisher's exact test. ${ }^{*}$ Mann-Whitney test. ${ }^{* *}$ Kruskal-Wallis test.

age, smoking, male sex, and underlying diseases such as chronic kidney disease, chronic obstructive pulmonary disease (COPD), and cerebrovascular disease are reportedly associated with higher disease severity ${ }^{11,12}$. Higher levels of serum biomarkers including lactate dehydrogenase (LDH), C-reactive protein (CRP), and D-dimer, as well as decreased blood platelet and lymphocyte counts have also been associated with more lethal conditions ${ }^{13}$. However, most of our knowledge regarding COVID-19 comes from Chinese studies and there is little known about the clinical and paraclinical findings of the patients in other regions. Our study aims to investigate further demographic, clinical, laboratory, and radiologic findings to design a protocol in order to assess the condition, prognosis, and response to treatment of COVID-19 infected inpatients in Iran.

\section{Results}

Demographic data and comorbid conditions. Overall, 200 patients were enrolled in the study, of whom 118 (59\%) were male and 82 (41\%) were female. Mean age of the patients was $69.75 \pm 6.39$ years. Disease was mild/moderate in 33 cases $(16.5 \%)$, severe in $139(69.5 \%)$, and critical in $28(14 \%)$. There was no significant difference regarding gender between the three groups of patients. Critical cases were significantly older compared with the mild/moderate and severe groups $(\mathrm{P}=0.009)$. However, there was no significant difference in the mean age when comparing the two groups of mild/moderate and severe/critical $(\mathrm{P}=0.149)$. Table 1 compares the demographic data and comorbid conditions in patients with different degrees of disease severity. Among all comorbid conditions, only malignancy had significantly different frequencies between the three groups $(\mathrm{P}<0.001)$.

Vital signs, clinical symptoms, and morbidities. Regarding the vital signs, pulse rate, respiratory rate, and oxygen saturation were significantly associated with the severity of disease, both in two-group and threegroup comparisons $(\mathrm{P}<0.05$; Table 2$)$. The frequency of dyspnea was significantly higher in severe/critical group, while nausea/vomiting was significantly more common among the mild/moderate cases $(\mathrm{P}=0.023$ and 0.016 , respectively). Confusion was significantly more common among critical cases, compared to the mild/moderate and severe groups $(\mathrm{P}=0.043)$. Furthermore, most of COVID-19-associated morbidities including shock, sepsis, coagulopathy, acidosis, ARDS, ICU admission, and intubation were significantly more common among the critical cases, compared with mild/moderate and severe groups $(\mathrm{P}<0.001)$. 


\begin{tabular}{|c|c|c|c|c|c|}
\hline \multirow[b]{2}{*}{ Feature } & \multicolumn{3}{|l|}{ Disease severity } & \multirow[b]{2}{*}{$P_{1}$} & \multirow[b]{2}{*}{$\mathbf{P}_{2}^{* * *}$} \\
\hline & Mild/moderate $(\mathrm{N}=33)$ & Severe $(\mathrm{N}=139)$ & Critical $(\mathrm{N}=28)$ & & \\
\hline \multicolumn{6}{|l|}{ Vital signs } \\
\hline Pulse rate & $87.12 \pm 20.12$ & $94.71 \pm 26.64$ & $106.48 \pm 26.36$ & $0.003^{* *}$ & $<0.001$ \\
\hline Respiratory rate & $20.12 \pm 1.97$ & $26.64 \pm 6.87$ & $26.36 \pm 6.76$ & $<0.001^{* *}$ & $<0.001$ \\
\hline $\mathrm{BP}(\mathrm{mmHg})$ & $126.71 \pm 21.98$ & $126.74 \pm 15.42$ & $129.68 \pm 23.68$ & $0.478^{* *}$ & 0.467 \\
\hline Temperature $\left({ }^{\circ} \mathrm{C}\right)$ & $37.63 \pm 0.65$ & $37.65 \pm 0.66$ & $37.98 \pm 0.83$ & $0.742^{* *}$ & 0.084 \\
\hline $\mathrm{O}_{2}$ saturation $(\%)$ & $95.48 \pm 1.25$ & $87.80 \pm 6.37$ & $82.15 \pm 10.00$ & $<0.001^{* *}$ & $<0.001$ \\
\hline \multicolumn{6}{|l|}{ Symptoms } \\
\hline Fever & $23(69.7)$ & $101(72.7)$ & $19(67.9)$ & $0.802^{\star}$ & 0.849 \\
\hline Dyspnea & $24(72.7)$ & $123(88.5)$ & $24(85.7)$ & $0.023^{*}$ & 0.069 \\
\hline Nausea/vomiting & $11(33.3)$ & $22(15.8)$ & $4(14.3)$ & $0.016^{*}$ & 0.055 \\
\hline Cough & $29(87.9)$ & $129(92.8)$ & $25(89.3)$ & $0.414^{*}$ & 0.595 \\
\hline Diarrhea & $8(24.2)$ & $19(13.7)$ & $2(7.1)$ & $0.082^{\star}$ & 0.148 \\
\hline Conjunctivitis & $0(0.0)$ & $2(1.4)$ & $0(0.0)$ & $0.528^{*}$ & 0.642 \\
\hline Myalgia & $16(48.5)$ & 69 (49.6) & $15(53.6)$ & $0.849^{*}$ & 0.914 \\
\hline Arthralgia & $7(21.2)$ & $18(12.9)$ & $2(7.1)$ & $0.156^{*}$ & 0.261 \\
\hline Weakness & $16(48.5)$ & $65(46.8)$ & $14(50.0)$ & $0.901^{*}$ & 0.945 \\
\hline Abdominal pain & $1(3.0)$ & $6(4.3)$ & $0(0.0)$ & $0.872^{\star}$ & 0.519 \\
\hline Seizure & $0(0.0)$ & $1(0.7)$ & $0(0.0)$ & $0.656^{\star}$ & 0.802 \\
\hline Headache & $3(9.1)$ & $26(18.7)$ & $2(7.1)$ & $0.266^{*}$ & 0.164 \\
\hline Sore throat & $1(3.0)$ & 19 (13.7) & $2(7.1)$ & $0.109^{*}$ & 0.167 \\
\hline Chill & $10(30.3)$ & $28(20.1)$ & $7(25.0)$ & $0.240^{*}$ & 0.428 \\
\hline Hyposmia & $4(12.1)$ & $11(7.9)$ & $2(7.1)$ & $0.414^{*}$ & 0.710 \\
\hline Fatigue & $9(27.3)$ & $40(28.8)$ & $7(25.0)$ & $0.919^{*}$ & 0.916 \\
\hline Confusion & $0(0.0)$ & $2(1.4)$ & $3(10.7)$ & $0.517^{*}$ & 0.043 \\
\hline Rhinorrhea & $1(3.0)$ & $3(2.2)$ & $0(0.0)$ & $0.802^{*}$ & 0.681 \\
\hline \multicolumn{6}{|l|}{ Morbidities } \\
\hline Shock & $0(0.0)$ & $1(0.7)$ & $6(21.4)$ & $0.231^{*}$ & $<0.001$ \\
\hline Sepsis & $0(0.0)$ & $1(0.7)$ & $8(28.6)$ & $0.172^{*}$ & $<0.001$ \\
\hline ARDS & $0(0.0)$ & $1(0.7)$ & $27(96.4)$ & $0.011^{*}$ & $<0.001$ \\
\hline DHF & $0(0.0)$ & $1(0.7)$ & $0(0.0)$ & $>0.999^{*}$ & 0.802 \\
\hline ATN & $0(0.0)$ & $1(0.7)$ & $1(3.6)$ & $>0.999^{*}$ & 0.288 \\
\hline Coagulopathy & $0(0.0)$ & $0(0.0)$ & $1(3.6)$ & $>0.999^{*}$ & 0.046 \\
\hline Acidosis & $0(0.0)$ & $0(0.0)$ & $1(3.6)$ & $>0.999^{*}$ & 0.046 \\
\hline ICU admission & $0(0.0)$ & $0(0.0)$ & $14(50.0)$ & $0.085^{\star}$ & $<0.001$ \\
\hline Intubation & $0(0.0)$ & $0(0.0)$ & $23(82.1)$ & $0.023^{*}$ & $<0.001$ \\
\hline
\end{tabular}

Table 2. Vital signs, clinical symptoms, and morbidities in patients with different disease severities. $B P$ blood pressure, $A R D S$ acute respiratory distress syndrome, $D H F$ diastolic heart failure, $A T N$ acute tubular necrosis, ICU intensive care unit. $\mathrm{P}_{1}$ : Comparison between mild/moderate and severe/critical groups. $\mathrm{P}_{2}$ : Comparison between mild/moderate, severe, and critical groups. Bold values are statistically significant $(\mathrm{P}<0.05) .{ }^{*} \mathrm{Chi}-$ square or Fisher's exact test. ${ }^{\star \star}$ Mann-Whitney test. ${ }^{\star * *}$ Kruskal-Wallis test.

Paraclinical data. Table 3 details the paraclinical findings of patients. As shown in the table, CRP, AST, pH, and $\mathrm{pO}_{2}$ were significantly associated with disease severity in three-group comparisons $(\mathrm{P}<0.05)$. However, twogroup comparison showed that only CRP and $\mathrm{pO}_{2}$ were significantly associated with disease severity $(\mathrm{P}<0.05$; Table 3). Chest CT showed that the extent of both ground-glass and consolidative pulmonary involvements were significantly higher in the critical and/or severe cases, compared with mild/moderate ones, in both two- and three-group comparisons $(\mathrm{P}<0.01)$. Severity of COVID-19 was not significantly associated with pleural effusion and bronchiectasis in chest CT.

Multivariate analyses. Multivariate logistic regression showed that $\mathrm{O}_{2}$ saturation, nausea/vomiting, and lung involvement of $\geq 50 \%$ in chest CT were independent predictors of severe/critical COVID-19. Whereas, only $\mathrm{O} 2$ saturation showed a significant association with severity when exclusively considering critical patients in multivariate analyses (Table 4). However, none of the assessed variables were significantly associated with ICU admission in a multivariate regression model.

Receiver operating characteristic (ROC) curve analyses. Our multivariate analyses have shown the level of $\mathrm{O}_{2}$ saturation to be strongly associated with the severity of COVID-19. ROC curve and area under ROC 


\begin{tabular}{|c|c|c|c|c|c|c|}
\hline \multirow[b]{2}{*}{ Feature } & & \multicolumn{3}{|l|}{ Disease severity } & \multirow[b]{2}{*}{$\mathbf{P}_{1}$} & \multirow[b]{2}{*}{$\mathbf{P}_{2}^{* * *}$} \\
\hline & & Mild/moderate $(\mathrm{N}=33)$ & Severe $(\mathrm{N}=139)$ & Critical $(\mathrm{N}=28)$ & & \\
\hline \multicolumn{7}{|l|}{ Laboratory findings } \\
\hline \multicolumn{2}{|l|}{ Lymphopenia } & $24(75.0)$ & $99(76.2)$ & $19(70.4)$ & $0.985^{*}$ & 0.818 \\
\hline \multicolumn{2}{|l|}{ VBG pH } & $7.42 \pm 0.05$ & $7.41 \pm 0.05$ & $7.10 \pm 1.29$ & $0.572^{* *}$ & 0.030 \\
\hline \multicolumn{2}{|l|}{ VBG $\mathrm{pO}_{2}(\mathrm{mmHg})$} & $36.27 \pm 9.11$ & $32.21 \pm 9.48$ & $31.85 \pm 8.51$ & $0.037^{\star \star}$ & 0.021 \\
\hline \multicolumn{2}{|l|}{ VBG pCO $\mathrm{pCO}_{2}(\mathrm{mmH})$} & $39.01 \pm 6.81$ & $40.33 \pm 7.84$ & $35.12 \pm 7.92$ & $0.824^{* *}$ & 0.112 \\
\hline \multicolumn{2}{|l|}{ VBG $\mathrm{pHCO}_{3}(\mathrm{mmHg})$} & $25.86 \pm 4.05$ & $25.94 \pm 5.05$ & $23.14 \pm 4.32$ & $0.705^{* *}$ & 0.054 \\
\hline \multicolumn{2}{|l|}{ AST (IU/L) } & $57.92 \pm 76.53$ & $48.55 \pm 74.94$ & $54.42 \pm 31.50$ & $0.470^{* *}$ & 0.031 \\
\hline \multicolumn{2}{|l|}{ ALT (IU/L) } & $59.64 \pm 87.35$ & $50.94 \pm 77.99$ & $49.78 \pm 60.37$ & $0.210^{* *}$ & 0.384 \\
\hline \multicolumn{2}{|l|}{$\mathrm{LDH}(\mathrm{U} / \mathrm{L})$} & $560.20 \pm 200.34$ & $660.74 \pm 434.76$ & $897.70 \pm 287.47$ & $0.310^{* *}$ & 0.137 \\
\hline \multicolumn{2}{|l|}{$\mathrm{CRP}(\mathrm{mg} / \mathrm{L})$} & $51.64 \pm 52.07$ & $86.22 \pm 62.33$ & $167.59 \pm 146.86$ & $0.003^{* *}$ & 0.001 \\
\hline \multicolumn{7}{|l|}{ Chest CT findings } \\
\hline \multirow{5}{*}{ Consolidation } & None & $8(24.2)$ & $36(25.9)$ & $5(17.9)$ & \multirow{5}{*}{$0.009^{*}$} & \multirow{5}{*}{0.002} \\
\hline & 1 lobe & $1(3.0)$ & $3(2.2)$ & $0(0.0)$ & & \\
\hline & 2 lobes & $21(63.6)$ & 55 (39.6) & $6(21.4)$ & & \\
\hline & 3 lobes & $0(0.0)$ & $1(0.7)$ & $2(7.1)$ & & \\
\hline & 4 lobes & $3(9.1)$ & $44(31.7)$ & $15(53.6)$ & & \\
\hline \multirow{5}{*}{ GGO } & None & $7(21.2)$ & $32(23.0)$ & $9(32.1)$ & \multirow{5}{*}{$<0.001^{*}$} & \multirow{5}{*}{$<0.001$} \\
\hline & 1 lobe & $3(9.1)$ & $0(0.0)$ & $0(0.0)$ & & \\
\hline & 2 lobes & $13(39.4)$ & $25(18.0)$ & $6(3.6)$ & & \\
\hline & 3 lobes & $0(0.0)$ & $4(2.9)$ & $0(0.0)$ & & \\
\hline & 4 lobes & $10(30.3)$ & $78(56.1)$ & $18(64.3)$ & & \\
\hline \multicolumn{2}{|l|}{$\mathrm{PE}$} & $1(3.0)$ & $8(5.8)$ & $4(14.3)$ & $0.376^{\star}$ & 0.168 \\
\hline \multicolumn{2}{|l|}{ Bronchiectasis } & $0(0.0)$ & $1(0.7)$ & $0(0.0)$ & $0.656^{*}$ & 0.802 \\
\hline
\end{tabular}

Table 3. Paraclinical data in patients with different disease severities. VBG venous blood gas, AST aspartate aminotransferase, $A L T$ alanine aminotransferase, $L D H$ lactate dehydrogenase, $C R P C$-reactive protein, $C T$ computed tomography, GGO ground-glass opacity, $P E$ pleural effusion. $\mathrm{P}_{1}$ : Comparison between mild/ moderate and severe/critical groups. $\mathrm{P}_{2}$ : Comparison between mild/moderate, severe, and critical groups. Bold values are statistically significant $(\mathrm{P}<0.05) .{ }^{\star}$ Chi-square or Fisher's exact test. ${ }^{*}$ Mann-Whitney test.

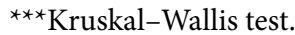

\begin{tabular}{|c|c|c|c|c|}
\hline \multirow[b]{2}{*}{ Predictor } & \multirow[b]{2}{*}{ Odds ratio } & \multicolumn{2}{|c|}{ 95\% confidence interval } & \multirow[b]{2}{*}{$\mathbf{P}$} \\
\hline & & Lower bound & Upper bound & \\
\hline \multicolumn{5}{|c|}{ Severe or critical disease } \\
\hline $\mathrm{O}_{2}$ saturation & 0.342 & 0.146 & 0.800 & 0.013 \\
\hline Nausea/vomiting & 45.937 & 1.513 & 1395.069 & 0.028 \\
\hline CT involvement $\geq 50 \%$ & 25.483 & 1.148 & 565.455 & 0.041 \\
\hline \multicolumn{5}{|l|}{ Critical disease } \\
\hline $\mathrm{O}_{2}$ saturation & 0.906 & 0.824 & 0.997 & 0.043 \\
\hline
\end{tabular}

Table 4. Multivariate regression for prediction of severe COVID-19.

curve (AUC) were used to determine the cut-off points of $\mathrm{O}_{2}$ saturation that can identify severe/critical and critical COVID-19.

ROC curves showed excellent diagnostic value of $\mathrm{O}_{2}$ saturation to identify severe/critical cases (AUC $=0.940$, 95\% CI 0.907-0.973, P<0.001; Fig. 1). The best cut-off point was $93.5 \%$, which yielded a sensitivity of $100 \%$ and a specificity of $90.3 \%$.

When considering only critical cases of COVID-19, $\mathrm{O}_{2}$ saturation showed an acceptable diagnostic value to identify these cases ( $\mathrm{AUC}=0.735,95 \% \mathrm{CI} 0.625-0.844, \mathrm{P}<0.001$; Fig. 2). The best cut-off point for $\mathrm{O}_{2}$ saturation in this regard was $80.5 \%$, which yielded a sensitivity of $89.5 \%$ and a specificity of $46.2 \%$.

\section{Discussion}

COVID-19 infection often causes a mild or even asymptomatic disease; however, some patients may proceed to severe and critical condition. Various clinical and paraclinical factors have been associated with higher disease severity ${ }^{14}$. However, most of the studies are from a restricted geographical region and there is a paucity of evidence regarding the determinant factors of poor prognosis in different ethnicities, as the features of COVID19 might differ in patients with different characteristics. We reviewed the clinical, laboratory, and imaging 


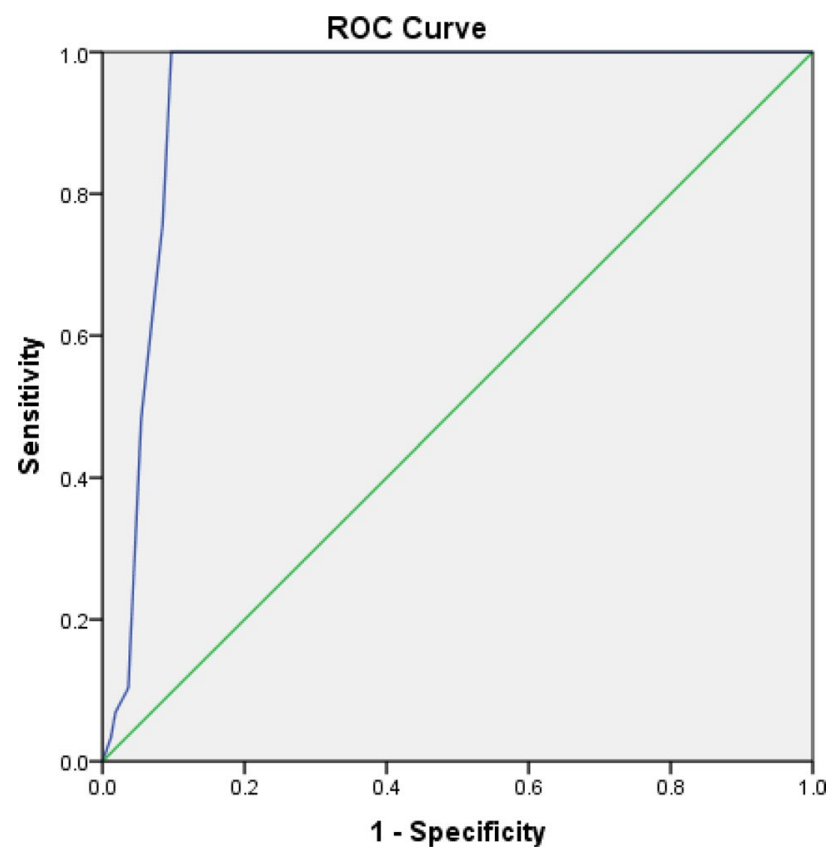

Figure 1. Receiver operating characteristic (ROC) curve of $\mathrm{O}_{2}$ saturation for identifying severe/critical COVID-19 infection.

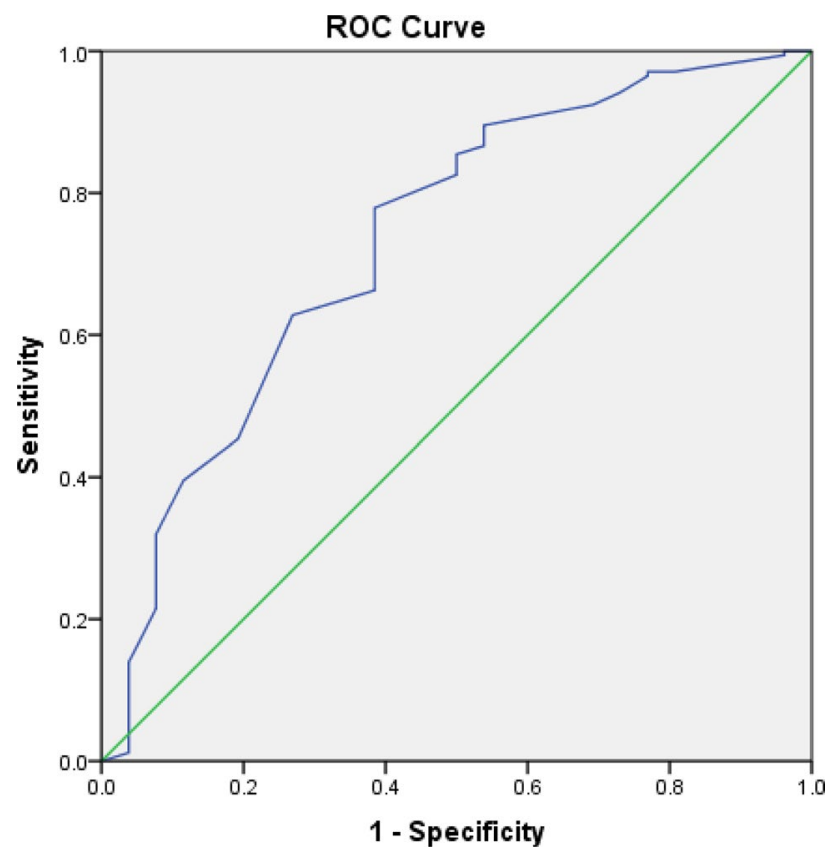

Figure 2. Receiver operating characteristic (ROC) curve of $\mathrm{O}_{2}$ saturation for identifying critical COVID-19 infection.

characteristics of COVID-19 inpatients in a tertiary center in Iran and assessed the factors that might possibly be associated with disease severity.

We found that critical cases of COVID-19 were significantly older compared to patients with lower severity. Malignant comorbidities were found to be considerably higher in critical and severe cases. Among vital signs, pulse rate, respiratory rate, and oxygen saturation were significantly associated with the severity of disease. Among symptoms, dyspnea, confusion, and nausea/vomiting were associated with higher disease severities. COVID-19-associated complications including shock, sepsis, coagulopathy, acidosis, ARDS, ICU admission, and intubation were significantly more common among the critical cases. Paraclinical factors that were associated with higher disease severity were increased CRP and AST, as well as decreased $\mathrm{pH}$ and $\mathrm{pO}_{2}$. Multivariate analyses 
showed that $\mathrm{O}_{2}$ saturation, nausea/vomiting, and $\geq 50 \%$ lung involvement in CT were independent predictors of severe COVID-19. O2 saturation was the sole independent predictor of critical condition in COVID-19 patients. The optimal cut-off values of $\mathrm{O}_{2}$ saturation for identifying severe/critical and critical disease were determined at $93.5 \%$ and $80.5 \%$, respectively.

In line with the findings of our study, several studies proposed that malignancy is associated with higher COVID-19 severity and poorer outcomes ${ }^{15-17}$. Therefore, it has been proposed that continuing antitumor treatment may further help the outcome of these patients ${ }^{15}$. Specifically, anti-tumor therapy is recommended to be continued in COVID-19 patients with acute leukemia, while for those with low grade malignancies, anti-cancer treatment is better to be postponed during the acute phase of infection ${ }^{18}$.

Although we found higher prevalence of diabetes, hypertension, and cardiovascular diseases among patients with severe disease compared with the mild/moderate group, this difference turned out to be statistically insignificant. This finding is inconsistent with the available literature that indicates a clear link between severity of COVID-19 and comorbidities such as COPD, diabetes, hypertension, and cardiovascular diseases ${ }^{19-21}$. This inconsistency is probably due to the relatively small sample of our study; a larger sample would have yielded statistically significant results, in line with previously reported findings. Moreover, previous studies mostly included Chinese population which may be another factor contributing to the incongruence between their outcomes and the results of our study.

As expected, we observed considerably lower $\mathrm{O}_{2}$ saturation and higher values of respiratory and pulse rate in patients with higher severities of the disease. It seems that the pulmonary involvement of COVID-19 and the subsequent respiratory distress, impairs cardiopulmonary functions causing a ventilation-perfusion mismatch ${ }^{22}$, which in turn leads to development of tachypnea and tachycardia. On the other hand, tachycardia can be related to fever in these patients ${ }^{23}$. However, we found no notable difference in the frequency of fever between patients with different disease severities.

AST was also found to be notably higher in severe COVID-19 infection in our patients. It might be hypothesized that the higher rate of hypoxia in more severe stages of the disease may contribute to liver injury and subsequent enzyme release as it is evident with AST release. However, the direct invasion of the virus to hepatocytes can also be proposed as an etiologic factor, which was reported by some studies ${ }^{24}$. Han et al. reported that AST could be an independent risk factor for more severe COVID-19 infections ${ }^{25}$, which was not the case in our multivariate assessments.

Another important finding of our study was the markedly higher level of CRP in patients with severe and critical COVID-19 disease. This factor is reported to be independently related to disease severity; CRP levels $>37.3 \mathrm{mg} / \mathrm{L}$ have been reportedly associated with poorer outcomes ${ }^{25}$. In our study, the serum level of CRP showed an incremental increase with the rise in disease severity from mild/moderate to critical. Consistently, Wang et al. reported that higher CRP levels were associated with higher lung involvement and more severe diseases ${ }^{26}$.

Our results indicate that higher severities of COVID-19 are associated with higher rates of serious complications such as shock, sepsis, ARDS, intubation, coagulopathy, and acidosis, which require ICU admission. It is generally believed that most of the COVID-19 cases develop mild to moderate symptoms and do not need hospitalization or ICU admission. However, some of them may need hospitalization and even intensive care. These patients are more likely to develop sepsis, shock, ARDS, and eventually death ${ }^{27}$. Furthermore, despite the usual presence of thrombocytopenia, coagulopathy is predictable in COVID-19 infected patients. Studies have reported elevated levels of D-dimer and higher frequency of thrombotic events in these patients, which might be related to inflammatory processes ${ }^{28}$. Acidosis can be present in some of the COVID-19 patients, which heralds a more severe stage of the disease ${ }^{7}$. In the present study, we found markedly lower $\mathrm{pH}$ levels in the venous blood gas (VBG) of patients with critical conditions, compared to other groups. This implies that acidosis is significantly associated with higher severities of the disease.

We found that low $\mathrm{O}_{2}$ saturation was the only independent predictor of critical condition and poor prognosis in COVID-19 patients. Higher $\mathrm{O}_{2}$ saturation was linked to a one-third lower risk for developing severe/critical disease. In line with our findings, a recent study on 167 patients in Anhui, China, reported that fingertip oxygen saturation and decreased CD4 cell count were the only independent predictors of severe COVID-1929.

We also found that the extent and severity of lung involvement on CT scan, as the number of involved lobes with consolidation or ground-glass opacification, was a significant and independent predictor of severe/critical COVID-19 infection. Similarly, Chaganti et al. developed a score for lung involvement that was composed of the number of lobes with consolidation or ground-glass opacification and found that this score was positively correlated with severe stages of COVID- $19^{30}$.

Among all symptoms, nausea/vomiting proved to be an independent predictive factor for severe disease and poorer prognosis. Several studies have indicated that gastrointestinal manifestations, namely nausea and vomiting, are common among COVID-19 patients. However, nausea and vomiting have not been alluded to as risk factors for severe conditions in these patients ${ }^{31,32}$.

A recent systematic review and meta-analysis on 1813 COVID-19 patients showed that dyspnea, COPD, cardiovascular diseases, and hypertension were predictive factors for severe COVID-19 disease and ICU admission ${ }^{33}$. A recent study on 548 patients from Wuhan indicated that older age, comorbid hypertension, high $\mathrm{LDH}$, and D-dimer were significantly associated with higher severity in cases with COVID-19 ${ }^{34}$. LDH was also identified as a risk factor for severe disease in another retrospective study of 47 patients from Wuhan, which also indicated lymphocyte count, especially CD3, CD4, and CD8 cells, as a predictive factor for higher severity ${ }^{35}$. Although age was significantly related to disease severity in our study, inconsistent with the mentioned studies, our multivariate analyses did not find significant associations between disease severity and age, comorbid conditions, LDH, and lymphocyte count. 
Our study can provide insights into the factors associated with higher risk for developing severe and critical COVID-19 infections in the Iranian population. The present study had some limitations. First of all, we had limited access to RT-PCR testing and could not perform it for all patients. Second, further survival and prognosis analyses was not performed, which may be applicable for further studies. Finally, when compared to the number of infected cases in Iran, our sample is relatively small.

In conclusion, $\mathrm{O}_{2}$ saturation, nausea/vomiting, and extent of lung involvement in chest CT can be potential factors that contribute to early prediction of severe and critical conditions in COVID-19 patients. It is therefore recommended to further evaluate the role of these factors in diagnosis and prognosis of patients with COVID19 in future studies.

\section{Methods}

Study settings and design. This cross-sectional study was conducted in Imam Reza hospital in Mashhad, the second largest city of Iran, during February-April 2020. With around 900 ward beds and 100 ICU beds, Imam Reza hospital, which is a tertiary center, is recognized as the main public center for COVID-19 patients in Mashhad, Iran. During the COVID-19 pandemic, approximately 400 ward beds and 40 ICU beds were exclusively assigned to COVID-19 inpatients.

Ethical approval. All enrolled patients provided informed written consent before entrance in to the study. Patients' data were kept coded without names and confidentiality was observed. The study was performed in accordance with the ethical codes of Helsinki declaration and was approved by the Ethics Committee of Mashhad University of Medical Sciences (approval code: IR.MUMS.REC.1398.308).

Study population. Patients with confirmed diagnoses of COVID-19 according to a positive RT-PCR and/ or typical chest computed tomography (CT) findings were included. The patients were classified based on the severity of disease according to the criteria proposed by $\mathrm{WHO}^{36}$, into the following groups:

- Mild/moderate with no or mild pneumonia;

- Severe with dyspnea (respiratory rate $>30)$ or hypoxia $\left(\mathrm{O}_{2}\right.$ saturation $\left.<93 \%\right)$;

- Critical with respiratory failure, shock, or multi-organ dysfunction.

Data collection. In order to gather patients' data, we designed a checklist according to the standards of reporting COVID-19 cases, proposed by $\mathrm{WHO}^{37}$. The checklist was discussed in a group of internal medicine specialists and subspecialists using a focus group technique to optimize the list by removing/adding some items.

Demographic data including age and gender, as well as medical and social history were recorded. In addition, clinical symptoms and vital signs were evaluated and recorded. Complications and outcomes including shock, sepsis, acute respiratory distress syndrome (ARDS), diastolic heart failure (DHF), acute tubular necrosis (ATN), intensive care unit (ICU) admission, intubation, coagulopathy, and acidosis were evaluated.

All patients were evaluated for pulmonary involvement using chest CT. The CT scans were then evaluated in terms of presence of abnormalities, mainly ground glass opacities (GGO), consolidation, pleural effusion, and bronchiectasis. The extent of lung involvement on CT scans were also determined based on the number of lobes involved. We considered those with GGO or consolidation in $\geq 3$ lobes in the chest CT as having $\geq 50 \%$ chest CT involvement.

Blood samples were taken and analyzed for several biomarkers. Laboratory findings including lymphocyte count and serum levels of aspartate aminotransferase (AST), alanine aminotransferase (ALT), LDH, and CRP levels, as well as blood gas analysis were assessed and recorded.

Statistical analysis. All the analyses were performed using SPSS (version 23 for Windows; IBM Statistics, Chicago, IL). Kolmogorov-Smirnov test was used to assess the normality of data. We made comparisons between all the three groups as well as between mild/moderate and severe/critical groups (merging the severe and critical cases). Chi-square test, Fisher's exact test, Mann-Whitney test, and Kruskal-Wallis test were used to compare data between different subgroups of patients. $\mathrm{P}<0.05$ was considered statistically significant in all tests.

Multivariate binary logistic regression models were used to assess the factors associated with disease severity and adverse outcomes. Odds ratio (OR) along with 95\% confidence interval (95\% CI) were used to report the results.

In order to determine the cut-off points of $\mathrm{O} 2$ saturation that can differentiate severe/critical and critical disease, ROC curve analyses were performed. Area under the ROC curve was used to confirm the strength of the prediction and optimal cut-off points were selected using the Youden index.

Received: 25 July 2020; Accepted: 6 April 2021

Published online: 22 April 2021

\section{References}

1. Chan, J.F.-W. et al. A familial cluster of pneumonia associated with the 2019 novel coronavirus indicating person-to-person transmission: A study of a family cluster. The Lancet 395, 514-523 (2020).

2. Holshue, M. L. et al. First case of 2019 novel coronavirus in the United States. N. Engl. J. Med. 382, 929 (2020).

3. Arabi, Y. M. et al. Middle east respiratory syndrome. N. Engl. J. Med. 376, 584-594 (2017).

4. Peiris, J. S., Yuen, K. Y., Osterhaus, A. D. \& Stöhr, K. The severe acute respiratory syndrome. N. Engl. J. Med. 349, 2431-2441 (2003). 
5. Statista. Infection Rates of Viruses Involved in Outbreaks Worldwide as of 2020 (2020). https://www.statista.com/statistics/1103196/ worldwide-infection-rate-of-major-virus-outbreaks/. Accessed 10 Mar 2021.

6. Mahase, E. Coronavirus covid-19 has killed more people than SARS and MERS combined, despite lower case fatality rate. BMJ. 368, m641 (2020).

7. Huang, C. et al. Clinical features of patients infected with 2019 novel coronavirus in Wuhan, China. The Lancet 395, 497-506 (2020).

8. Bai, T. et al. Clinical and Laboratory factors predicting the prognosis of patients with COVID-19: An analysis of 127 patients in Wuhan, China. SSRN J. https://doi.org/10.2139/ssrn.3546118 (2020).

9. Liu, J. et al. Exploring the law of development and prognostic factors of common and severe COVID-19: A retrospective casecontrol study in 122 patients with complete course of disease. SSRN J. https://doi.org/10.2139/ssrn.3555209 (2020).

10. Zhou, F. et al. Clinical course and risk factors for mortality of adult inpatients with COVID-19 in Wuhan, China: A retrospective cohort study. The Lancet 395, 1054 (2020).

11. Rathore, V., Galhotra, A., Pal, R. \& Sahu, K. K. COVID-19 pandemic and children: A review. J. Pediatr. Pharmacol. Therap. 25, 574-585. https://doi.org/10.5863/1551-6776-25.7.574 (2020).

12. Mishra, A. K., Sahu, K. K., George, A. A., Sargent, J. \& Lal, A. Cerebrovascular events in COVID-19 patients. Monaldi Arch. Chest Dis. https://doi.org/10.4081/monaldi.2020.1341 (2020).

13. Zhao, X. et al. Incidence, clinical characteristics and prognostic factor of patients with COVID-19: A systematic review and metaanalysis. MedRxiv. https://doi.org/10.1101/2020.03.17.20037572v1 (2020).

14. Verity, R. et al. Estimates of the severity of COVID-19 disease. MedRxiv. https://doi.org/10.1101/2020.03.09.20033357 (2020).

15. Zhang, L. et al. Clinical characteristics of COVID-19-infected cancer patients: A retrospective case study in three hospitals within Wuhan, China. Ann. Oncol. 31, 838 (2020).

16. Oh, W. K. COVID-19 infection in cancer patients: Early observations and unanswered questions. Ann. Oncol. 31, 838 (2020).

17. Ma, J., Yin, J., Qian, Y. \& Wu, Y. Clinical characteristics and prognosis in cancer patients with COVID-19: A single center's retrospective study. J. Infect. 81, 318 (2020).

18. Karunakaran, P., Nampoothiri, R. V. \& Sahu, K. K. Managing blood disorders during the Covid-19 pandemic: Current pharmacological insights. Expert Rev. Clin. Pharmacol. 13, 1285-1287. https://doi.org/10.1080/17512433.2020.1841633 (2020).

19. de Almeida-Pititto, B. et al. Severity and mortality of COVID 19 in patients with diabetes, hypertension and cardiovascular disease: A meta-analysis. Diabetol. Metab. Syndr. 12, 75. https://doi.org/10.1186/s13098-020-00586-4 (2020).

20. Jain, V. \& Yuan, J.-M. Predictive symptoms and comorbidities for severe COVID-19 and intensive care unit admission: A systematic review and meta-analysis. Int. J. Public Health 65, 533-546. https://doi.org/10.1007/s00038-020-01390-7 (2020).

21. Luo, L. et al. The potential association between common comorbidities and severity and mortality of coronavirus disease 2019: A pooled analysis. Clin. Cardiol. 43, 1478-1493. https://doi.org/10.1002/clc.23465 (2020).

22. Gattinoni, L., Chiumello, D. \& Rossi, S. COVID-19 pneumonia: ARDS or not?. Crit. Care 24, 154 (2020).

23. Hui, H. et al. Clinical and radiographic features of cardiac injury in patients with 2019 novel coronavirus pneumonia. MedRxiv 51, $372(2020)$.

24. Zhang, C., Shi, L. \& Wang, F.-S. Liver injury in COVID-19: Management and challenges. Lancet Gastroenterol. Hepatol. 5, 428 (2020).

25. Han, Y. et al. Lactate dehydrogenase, a risk factor of severe COVID-19 patients. MedRxiv 19, 353 (2020).

26. Ling, W. C-reactive protein levels in the early stage of COVID-19. Med. et Maladies Infect. 50, 332 (2020).

27. Cascella, M., Rajnik, M., Cuomo, A., Dulebohn, S. C. \& Di Napoli, R. Statpearls (StatPearls Publishing, 2020).

28. Terpos, E. et al. Hematological findings and complications of COVID-19. Am. J. Hematol. https://doi.org/10.1002/ajh.25829 (2020).

29. Wei, Y. Y. et al. Risk factors for severe COVID-19: Evidence from 167 hospitalized patients in Anhui, China. J. Infect. https://doi. org/10.1016/j.jinf.2020.04.010 (2020).

30. Chaganti, S. et al. Quantification of Tomographic Patterns associated with COVID-19 from Chest CT. Preprint at http://arXiv.org/ 2004.01279 (2020).

31. Wong, S. H., Lui, R. N. \& Sung, J. J. Covid-19 and the digestive system. J. Gastroenterol. Hepatol. 35, 744-748 (2020).

32. Tian, Y., Rong, L., Nian, W. \& He, Y. gastrointestinal features in COVID-19 and the possibility of faecal transmission. Aliment. Pharmacol. Ther. 51, 843-851 (2020).

33. Jain, V. \& Yuan, J-M. Predictive symptoms and comorbidities for severe COVID-19 and intensive care unit admission: a systematic review and meta-analysis. Int. J. Public Health 65, 533-546 (2020).

34. Li, X. et al. Risk factors for severity and mortality in adult COVID-19 inpatients in Wuhan. J. Allergy Clin. Immuno. https://doi. org/10.1016/j.jaci.2020.04.006 (2020).

35. Han, Y. et al. Lactate dehydrogenase, a Risk Factor of Severe COVID-19 Patients: A Retrospective and Observational Study. MedRxiv. https://doi.org/10.1101/2020.03.24.20040162 (2020).

36. World Health Organisation. Clinical Management of Severe Acute Respiratory Infection When Novel Coronavirus (2019-nCoV) Infection is Suspected: Interim Guidance 21 (WHO, 2020).

37. World Health Organization. Clinical Management of Severe Acute Respiratory Infection (SARI) When COVID-19 Disease is Suspected: Interim Guidance, 13 March 2020 (World Health Organization, 2020).

\section{Acknowledgements}

This work was supported by the Vice Chancellor of Research, Mashhad University of Medical Sciences (Grant Number 981800).

\section{Author contributions}

S.N., Z.J., M.M., A.A., M.S., S.R. and M.A. conceived and planned the project. S.N., F.E., S.K. and N.S. carried out the project. R.B., S.H., S.N., F.R., M.A., M.M. and N.S. contributed to sample preparation. M.E., A.Z. and M.R. analyzed the data. M.R. and A.Z. wrote the manuscript with input from all authors. A.A., Z.J. and M.M. supervised the project. All authors provided critical feedback and helped shape the research, analysis and manuscript.

\section{Competing interests}

The authors declare no competing interests.

\section{Additional information}

Correspondence and requests for materials should be addressed to M.M.

Reprints and permissions information is available at www.nature.com/reprints. 
Publisher's note Springer Nature remains neutral with regard to jurisdictional claims in published maps and institutional affiliations.

(c) (i) Open Access This article is licensed under a Creative Commons Attribution 4.0 International License, which permits use, sharing, adaptation, distribution and reproduction in any medium or format, as long as you give appropriate credit to the original author(s) and the source, provide a link to the Creative Commons licence, and indicate if changes were made. The images or other third party material in this article are included in the article's Creative Commons licence, unless indicated otherwise in a credit line to the material. If material is not included in the article's Creative Commons licence and your intended use is not permitted by statutory regulation or exceeds the permitted use, you will need to obtain permission directly from the copyright holder. To view a copy of this licence, visit http://creativecommons.org/licenses/by/4.0/.

(C) The Author(s) 2021 